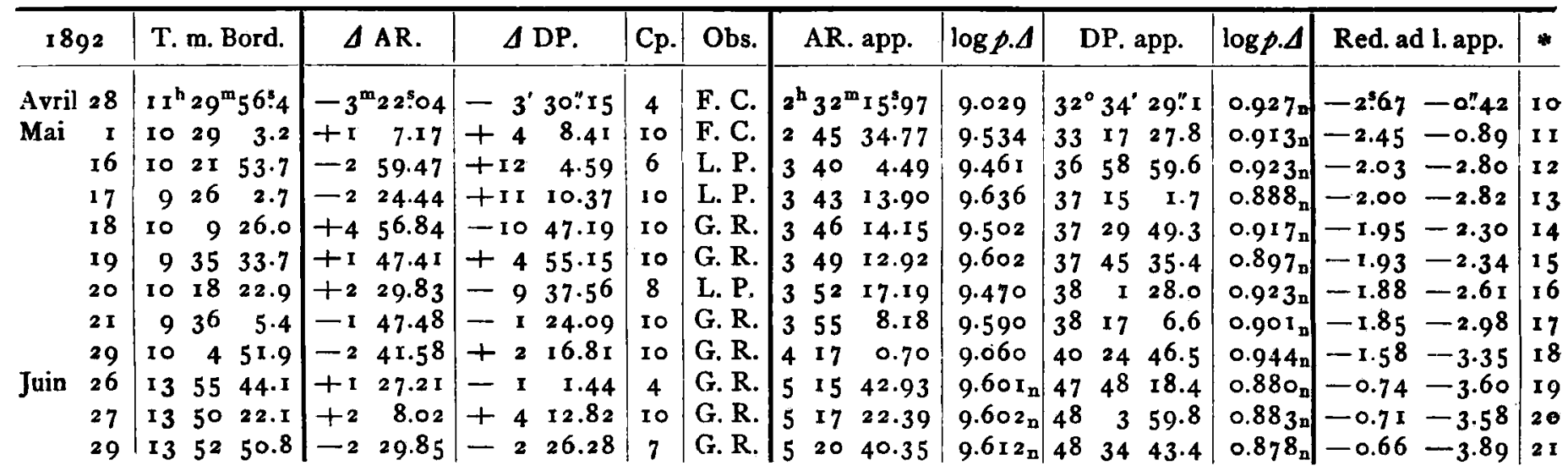

Positions moyennes des étoiles de comparaison.

\begin{tabular}{|c|c|c|c|}
\hline * & AR. 1892.0 & DP. 1892.0 & Autorité \\
\hline $\mathbf{I}$ & $23^{\mathrm{h}} 59^{\mathrm{m}} 31^{\mathrm{s}} \div 10$ & $29^{\circ} \times 7^{\circ} \times 4^{\prime \prime} 8$ & AG. Hels. 14657 \\
\hline 2 & $\circ 2034.59$ & 29 I I 9.8 & AG. Hels. 316 \\
\hline 3 & I 315.40 & $\begin{array}{lll}30 & 4 & 27.2\end{array}$ & $\begin{array}{l}1 / 2 \text { (AOe. 1 } 74 \mathrm{I}+\mathrm{AG} \text {. Hels. } \\
1413 \text { ) }\end{array}$ \\
\hline 4 & I 2854.66 & $3020 \quad 23.2$ & $\begin{array}{l}1 / 2(\mathrm{Ll} .2827+\text { AG. Hels. } \\
1368)\end{array}$ \\
\hline 5 & $147 \quad 14.3^{\circ}$ & $\begin{array}{lll}30 & 4 I & 50.9\end{array}$ & $1 / 2$ (BB.VI + AG.Hels. 1674$)$ \\
\hline 6 & I 5048.57 & $30 \quad 54$ & $\begin{array}{l}1 / 2(\text { Ll. } 3536+\text { AG. Hels. } \\
\text { I 728) }\end{array}$ \\
\hline 7 & $2 \quad 5 \quad 29.07$ & $\begin{array}{lll}3 & 42 & 30.4\end{array}$ & $\begin{array}{l}1 / 2 \text { (AOe. } 2454+\text { AG. Hels. } \\
\text { I } 96 \text { I) }\end{array}$ \\
\hline 8 & $2 \quad 18 \quad 57.22$ & $\begin{array}{lll}31 & 36 & 45.8\end{array}$ & $\begin{array}{l}1 / 2 \text { (Ll. } 4405+\text { AG. Hels. } \\
2234)\end{array}$ \\
\hline 9 & $2 \quad 23 \quad 37.82$ & $\begin{array}{lll}32 & 27 & 21.8\end{array}$ & $\begin{array}{l}1 / 2 \text { (Ll. } 4543-5+\text { AG. Hels. } \\
\quad 229 \mathrm{I})\end{array}$ \\
\hline
\end{tabular}

\begin{tabular}{|c|c|c|c|c|c|}
\hline$*$ & AR. I & 392.0 & DP. & 1892.0 & Autorité \\
\hline 10 & $2^{h} 35$ & 40.68 & $32^{\circ}$ & $37^{\prime} 59^{\prime \prime} 6$ & $\begin{array}{l}1 / 2 \text { (AOe. } 3055^{-6}+\text { AG. } \\
\text { Hels. } 2459\end{array}$ \\
\hline I I & 244 & 30.05 & 33 & II 20.3 & $\begin{array}{l}1 / 2 \text { (AOe. } 3227+\text { AG. Hels. } \\
2568 \text { ) }\end{array}$ \\
\hline 12 & 343 & 3.99 & 36 & $4^{6} \quad 57.8$ & AOe. 4 I 8 I \\
\hline $\mathbf{1} 3$ & 345 & 40.34 & 37 & 354.1 & AOe. $4223-4$ \\
\hline 14 & $34 I$ & 19.26 & 37 & $40 \quad 38.8$ & Str. P.M. 377 \\
\hline 15 & 347 & 27.44 & 37 & $40 \quad 42.9$ & AOe. $4252-4$ \\
\hline I 6 & 349 & 49.24 & 38 & I 8.2 & AOe. $4283-4-5$ \\
\hline 17 & $35^{6}$ & 57.51 & 38 & $18 \quad 34.7$ & BB.VI $+51: 848$ \\
\hline 18 & 419 & 43.86 & 40 & 2233.0 & BB.VI $+49^{\circ} \times 183$ \\
\hline 19 & 514 & 16.46 & 47 & $49 \quad 23.4$ & BB.VI $+42: 1258$ \\
\hline 20 & 5 I 5 & $\mathrm{r} 5.08$ & 47 & 5950.6 & $W_{2} 5^{h} \cdot 34 I$ \\
\hline 21 & $5 \quad 23$ & 10.86 & & $37 \div 3.6$ & Lal. 10224 \\
\hline
\end{tabular}

Les observateurs sont: G. R. $=$ G. Rayet, L. P. $=$ L. Picart, F. C. $=$ F. Courty.

Bordeaux 1892 Sept. 4.

G. Rayet.

\title{
Beobachtungen der Cometen 1892 I und 1892 II
}

am 9 zölligen Refractor der Marine-Sternwarte in Nicolajew, von $\mathcal{F}$. Kortazzi.

\begin{tabular}{l|l|l|l|l|l|l|l|l|l|}
\hline 1892 & M.Z. Nicol. & $\Delta \alpha$ & $\Delta \delta$ & Vgl. & $\alpha$ app. & $\log p . \Delta$ & $\delta$ app. & $\log p . \Delta \mid$ Red. ad l. app. & $*$ \\
\hline
\end{tabular}

Comet $1892 \mathrm{I}$.

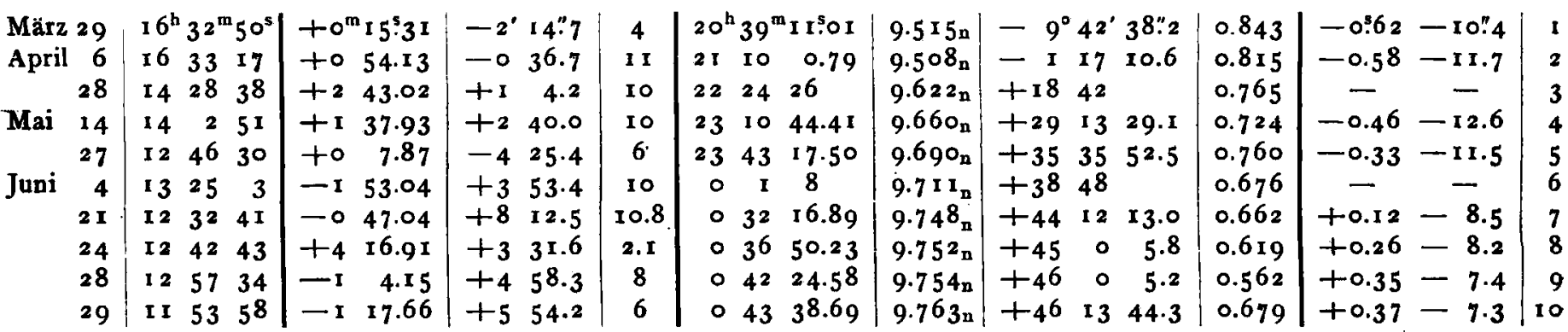

März 29-April 28. Die Beobachtungen sind bei beleuchtetem Feld angestellt. - Juni 24 . Die Beobachtungen wurden durch Wolken unterbrochen. - Die Verbesserungen wegen Refraction sind angebracht. 


\begin{tabular}{|c|c|c|c|c|c|c|c|c|c|c|}
\hline 1892 & M. Z. Nicol. & $\Delta \alpha$ & $\Delta \delta$ & Vgl. & $\alpha$ app. & $\log p \cdot \Delta$ & $\delta$ app. & $|\log p .4|$ & Red. ad l. app. & * \\
\hline
\end{tabular}

Comet r8g2 II.

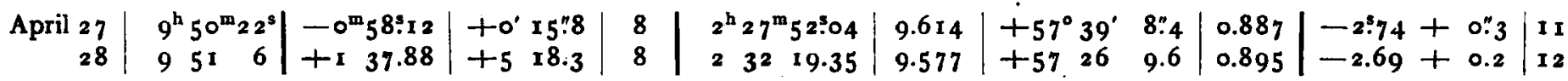

Mittlere Oerter der Vergleichsterne far 1892.0.

\begin{tabular}{|c|c|c|c|}
\hline * & $\alpha$ & $\delta$ & Autorităt \\
\hline I & $20^{\mathrm{h}} 3^{8^{m}} 5^{6^{s}} \cdot 3^{2}$ & $-9^{\circ} 40^{\circ}$ I $33^{\prime \prime}$ I & $\mathrm{M}_{1} 25^{8006}$ \\
\hline 2 & $2 \mathrm{I} \quad 9 \quad 7.24$ & - I 1645.6 & AG. Nicol. (Manusc.) \\
\hline 3 & $22 \quad 21 \quad 44$ & +1841 & BD. $+18: 4989$ \\
\hline 4 & $\begin{array}{lll}23 & 9 & 6.94\end{array}$ & $\begin{array}{lll}+29 & 11 & 1.7\end{array}$ & $W_{2} 23^{\mathrm{h}} \times 3 \mathrm{r}-2$ \\
\hline 5 & $\begin{array}{lll}23 & 43 & 9.96\end{array}$ & $+3540 \quad 29.4$ & AG. Lund \\
\hline 6 & 3 & +3844 & BD. +389 \\
\hline
\end{tabular}

Nicolajew 1892 Juli ro.

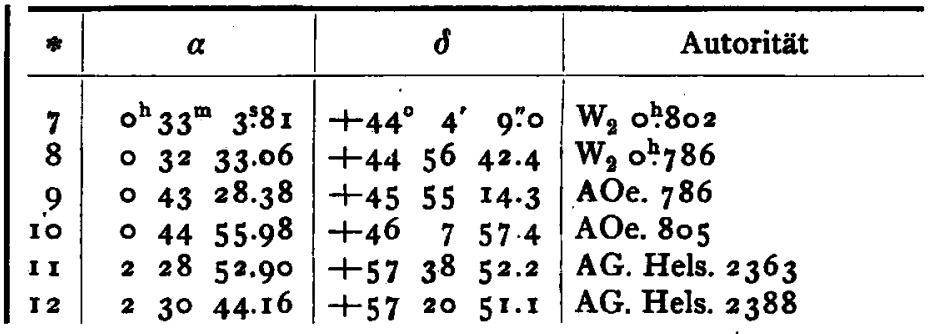

F. Kortazzi.

\section{Cordoba Observations of (71) Niobe.}

(Schreiben an den Herausgeber.)

I send you the results of the filar micrometer and meridian circle observations of Niobe which we were able to make at opposition. Those upon March 14, 15 and April 4 were made by me, and the remainder, including all the meridian observations, were made by Assistant Lester $C$. Taylor. The planet was of the 9.7 mag., which is near the limit of reach of our $\mathbf{M}$. C.

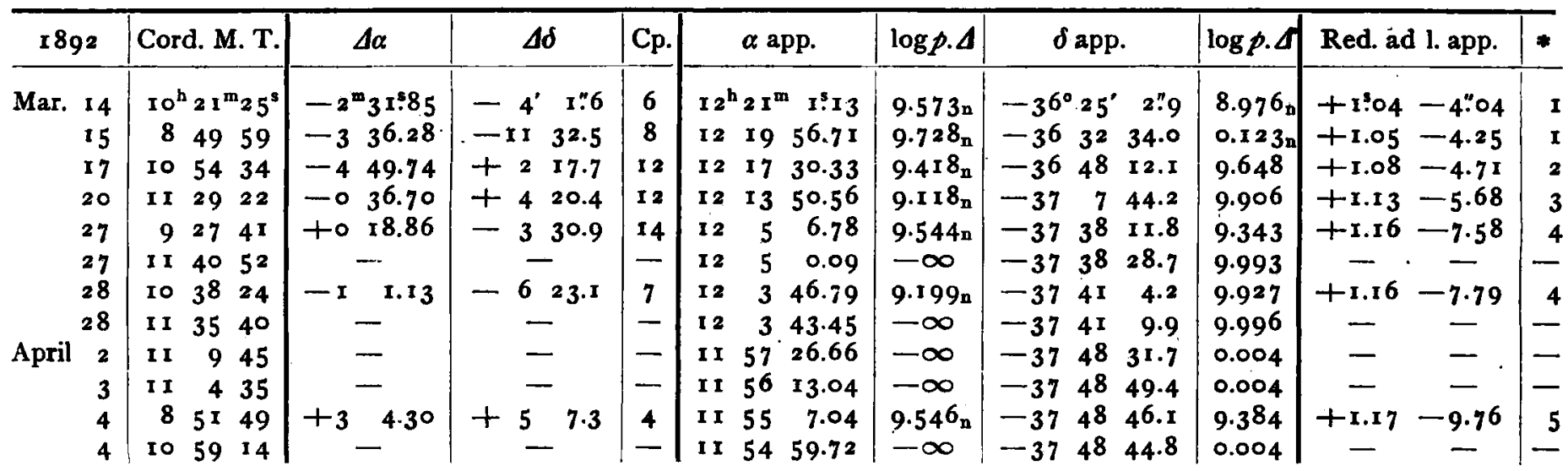

Mean Places of the Comparison Stars for 1892.0.

\begin{tabular}{|c|c|c|c|}
\hline * & $\alpha$ & $\delta$ & Authority \\
\hline $\mathbf{I}$ & $12^{\mathrm{h}} 23^{\mathrm{m}} 3^{1} \mathrm{I}^{\mathrm{s}} 94$ & $-3^{6^{\circ}} 20^{\prime} 57^{\prime \prime} \cdot 3$ & Cord. ZC. I 3 \\
\hline 2 & $1222 \quad 18.99$ & $-36 \quad 50 \quad 25.1$ & Cord. ZC. 1326 \\
\hline 3 & $12 \quad 14 \quad 26.13$ & $\begin{array}{lll}-37 & 1 \times 8.9\end{array}$ & Cord. GC. 168 I 5 \\
\hline 4 & I2 446.76 & $\begin{array}{lll}-37 & 34 & 33.3\end{array}$ & Cord. ZC. 240 \\
\hline$=$ & $\begin{array}{lll}1152 & 1.57\end{array}$ & $-3753 \quad 43.6$ & Cord. GC. $16_{320}$ \\
\hline
\end{tabular}

Argentine National Observatory, Cordoba, 1892 June 20.

Fohn M. Thome. 